\title{
Green Synthesis of Noble Metal of Platinum Nanoparticles from Ocimum sanctum (Tulsi) Plant- Extracts
}

\author{
N. Prabhu ${ }^{1}$, T. Gajendran ${ }^{2}$ \\ Department of Biotechnology, Bharathidasan Institute of Technology, Anna University, Tiruchirappalli-620 \\ 024, Tamilnadu, India
}

\begin{abstract}
The most effectively studied nanoparticles in the current past are those made from the noble metals such as silver, gold and platinum. Nanoparticles find vast applications in various fields ranging from medical to physical fields. To meet the increasing demands for commercial nanoparticles new eco-friendly "green" methods of synthesis are being discovered. Plant mediated synthesis of nanoparticles offers single step, easy extracellular synthesis of nanoparticles. The leaf extract of Ocimum sanctum was used as a reducing agent for the synthesis of platinum nanoparticles from an aqueous chloroplatinic acid $\left(\mathrm{H}_{2} \mathrm{PtCl}_{6} \cdot 6 \mathrm{H}_{2} \mathrm{O}\right)$. A greater conversion of platinum ions to nanoparticles was achieved by employing a Tulsi leaf broth with a reaction temperature of $100{ }^{\circ} \mathrm{C}$. The successful formation of platinum nanoparticles has been confirmed by UV-Vis spectro photometer, Fourier Transform Infrared Spectroscopy (FTIR), X-Ray Diffraction (XRD), Scanning Electron Microscopy (SEM) and Transmission Electron Microscopy (TEM). It is evident from scanning electron microscopy that the reduced platinum particles were found as aggregates with irregular shape. FTIR studies confirmed the presence of platinum nanoparticles which may responsible for reduction of platinum ions to platinum nanoparticles, yet further research is needed in this area to explore the possible biomolecules responsible for the bioreduction process. The XRD pattern revealed the complex crystalline nature of platinum nanoparticle. In this method there is no need to use high pressure, energy, temperature and toxic chemicals in case of chemical and physical method.
\end{abstract}

Keywords: Nanoparticle, Ocimum sanctum, UV-Vis spectro photometer, FTIR, XRD, SEM TEM

\section{Introduction}

The nanoscience and nanotechnology is the most active discipline all around the world and considered as the fastest growing technological-revolution of the human history has ever seen [1]. Using plants for nanoparticle synthesis can be advantageous over other biological processes because it eliminates the elaborate process of maintaining cell cultures and can also be suitably scaled up for large-scale nanoparticle synthesis [2] Nature has devised various processes for the synthesis of nano scale materials which have paved the way for the development of relatively new and largely unexplored area of research in the nanomaterial synthesis field. Currently, nanoparticles (NPs) have drawn marvellous consideration because of their valuable properties on various fields such as medical, sensor, optical, electronic, and catalytic application [3]. The synthesis of nanoparticles has become the matter of great interest in recent times due to its various advantageous properties and applications in various fields. Platinum is one of the rarest and most expensive metals. It has high corrosion resistance and numerous catalytic applications including automotive catalytic converters and petrochemical cracking catalysts. Platinum nanoparticles are usually used in the form of colloid or suspension in a fluid. synthesis of platinum nanoparticles was achieved by Song et al.[4] using the leaf extract of Diopyros kaki. They reported that more than $90 \%$ of the platinum ions were converted into nanoparticles using $10 \%$ leaf biomass concentration at $95{ }^{\circ} \mathrm{C}$, and the average size of synthesized nanoparticles ranged from 2 to $12 \mathrm{~nm}$. Coccia et al [5] reported one-pot synthesis of platinum and palladium nanoparticles using lignin isolated from red pine (Pinus resinosa). Rapid synthesis of platinum nanoparticles has also been reported by Soundarrajan et al. [6] Ocimum sanctum (Tulsi) is a medicinal herb abundantly found and cultured in India, Malaysia, Australia, West Africa, and some of the Arab countries [7]. Tulsi leaves have been traditionally used for treatment of many infections. The antibacterial activity has been reported to be the upshot of essential oil components, mostly eugenols found in it. The present study aims at the synthesis of platinum nanoparticles from the aqueous extract of Tulsi leaves. We also attempt to combine the inherent antimicrobial activities of platinum metal and Tulsi extract for enhanced antimicrobial activity.

\subsection{Sample Collection}

\section{Materials And Methods}

Fresh green and mature leaves of ocimum sanctum were collected from Madurai (Tamilnadu, India) and used for preparation of extract. Platinum (IV) chloride, $\mathrm{H}_{2} \mathrm{PtCl}_{6} \cdot 6 \mathrm{H}_{2} \mathrm{O}$ were purchased from Merck specialties Private Limited, Mumbai. 


\subsection{Preparation of Leaf Extract}

In general manner, $30 \mathrm{~g}$ of healthy and matured leaves of plants portion were selected and then washed thoroughly. These leaves were grinded well and collected using glass vessels. Afterwards, it was boiled in $100 \mathrm{ml}$ of de-ionized water for 30 minutes. The mixture was cooled and this extract is filtered by vacuum filter. This filtrate was used as the extract for the preparation of platinum nanoparticles.

\subsection{Synthesis of Platinum (Pt) Nanoparticles}

$100 \mathrm{ml}$ of $0.5 \mathrm{mM}, 1 \mathrm{mM}, 1.5 \mathrm{mM}$ and $2 \mathrm{mM}$ of metal solution of $\mathrm{H}_{2} \mathrm{PtCl}_{6}$ was prepared. And plant extract is added to the metal solution for the reduction of nanoparticles. $90 \mathrm{ml}$ of $1 \mathrm{mM} \mathrm{H}_{2} \mathrm{PtCl}_{6} .6 \mathrm{H}_{2} \mathrm{O}$ was stirred with magnetic stirrer. $10 \mathrm{ml}$ of prepared plant extract was added to the solution in the ratio of 1:9. Again, the mixture was stirred with magnetic stirrer for more than 20 minutes. The colour change (Brown colour) is the evidence for the bio reduction of the ions.

\subsection{Powdered Nanoparticle}

Synthesized nanoparticles were centrifuged at $10,000 \mathrm{rpm}$ for 15 minutes, which was followed by the lyophilisation procedures to obtain the powdered nanoparticle.

\subsection{Characterization of Synthesized Nanoparticle}

Characterization of the synthesized nanoparticle was done by UV-Vis spectrophotometer, Fourier Transform Infra Red Spectroscopy, X-Ray Diffraction and Scanning Electron Microscopy.

\subsubsection{UV- Visible spectrophotometer}

The reduction of the pure Pt ions was monitored by UV- Visible spectrophotometer by diluting a small aliquot of sample with distilled water. UV-Visible spectroscopic analysis was done using UV-Visible spectrophotometer.

\subsubsection{Fourier Transform Infra Red Spectroscopy (FT-IR Spectroscopy)}

The FT-IR spectrum is used to examine the bio molecules that are responsible for the bio reduction of ions and capping of bio reduced nanoparticle by the ocimum sanctum leaves. For FT-IR the nanosample was centrifuged and lyophilized to obtain in powdered form. Fourier transform infra red spectra of the nanosamples were measured using a Perkin-Elmer Spectrum.

\subsubsection{X-Ray Diffraction (XRD)}

The nanoparticle thus obtained was purified by centrifugation at $10000 \mathrm{rpm}$ for 15 minutes and the pellet is collected. After lyophilisation of the purified nano particles, the structure and composition were analyzed by $\mathrm{XRD}$ and SEM. The dried mixture of nanoparticles was collected for the determination of the formation of $\mathrm{Pt}$ nanoparticles by an X'Pert Pro x-ray diffractometer operated at a voltage of $40 \mathrm{kV}$ and a current of $30 \mathrm{~mA}$ with $\mathrm{Cu} \mathrm{K} \alpha$ radiation in $\theta-2 \theta$ configurations. The crystallite domain size was calculated from the width of the XRD peaks, assuming that they are free from non-uniform strains using the Scherrer formula.

$$
D=0.94 \lambda / \beta \operatorname{Cos} \theta
$$

Where

$\mathrm{D}$ is the average crystallite domain size perpendicular to the reflecting planes;

$\lambda$ is the $\mathrm{X}$-ray wavelength;

$\beta$ is the full width at half maximum (FWHM);

$\theta$ is the diffraction angle.

\subsubsection{Scanning Electron Microscope (SEM) analysis}

Scanning Electron Microscopic (SEM) analysis was done using Hitachi S-4500 SEM machine. Thin films of the sample were prepared on a carbon coated copper grid by just dropping a very small amount of the sample on the grid, extra solution was removed using a blotting paper and then the film on the SEM grid were allowed to dry by putting it under a mercury lamp for $5 \mathrm{~min}$.

\subsubsection{Transmission electron microscopy (TEM) analysis}

Samples for TEM studies were prepared by placing a drop of the platinum Nanoparticles colloidal suspension obtained by bio reduction on lacy carbon-coated grids.TEM observations were performed on a transmission electron microscope JEOL 2010. 


\subsection{Synthesis of Silver Nanoparticles}

\section{Results And Discussion}

$1 \mathrm{~mL}$ of ocimum sanctum leaf extract was added to $9 \mathrm{ml}$ of $2 \mathrm{mM} \mathrm{H}_{2} \mathrm{PtCl}_{6}$ solution. It was observed that the colour of the solution had changed from dark green to dark brown with black within few minutes of addition. This indicated the formation of platinum nanoparticles. The colour change was due to the excitation of surface Plasmon vibrations in the metal nanoparticles [9]. The visual observation of platinum nanoparticles formation is shown in Figure 1.

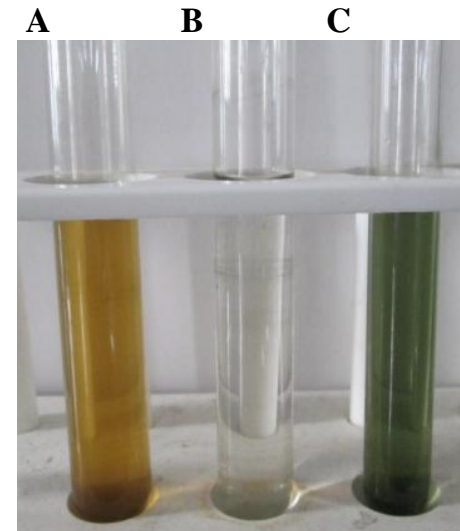

Figure 1 Visual observation of platinum nanoparticles synthesis (A- Reaction mixture, B-Salt solution, C- Plant extracts)

\subsection{UV-Visible Spectral Analysis}

The absorption spectrum of PtNPs formed in the reaction had a peak at $400 \mathrm{~nm}$ which confirms the presence of platinum nanoparticles (figure 2).

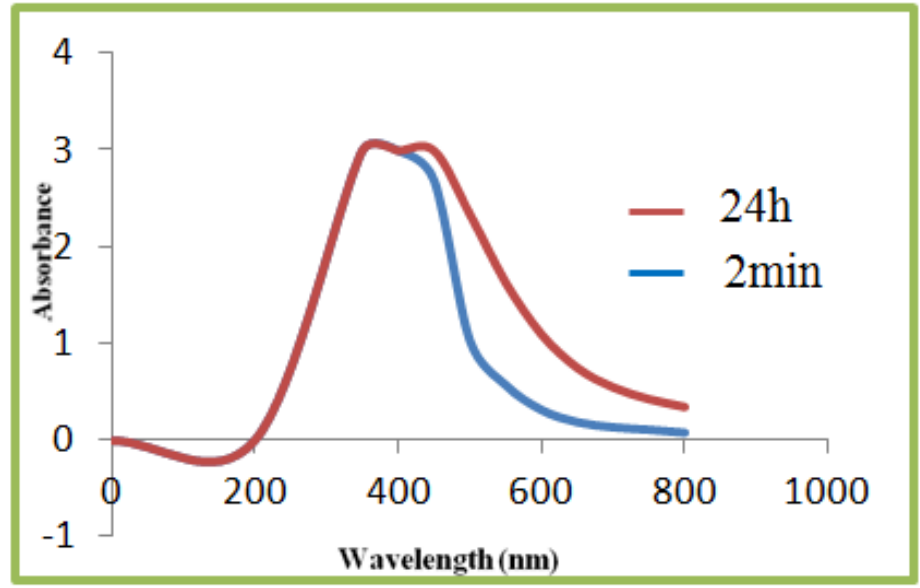

Figure 2 UV-Visible spectra of PtNPs at different incubation time

\subsection{Fourier Transform Infra Red Spectroscopy Analysis}

The FT-IR spectra were used to identify the possible bio molecules responsible for the reduction of the Pt ions and capping of the ocimum sanctum formed PtNPs.

Figure 3 shows the FTIR spectra of ocimum sanctum aqueous extract and bio-synthesized PtNPs. In the FTIR spectrum of ocimum sanctum the signal at $1251 \mathrm{~cm}-1$ corresponded to asymmetric stretching vibration of a sulphate and band at $1074 \mathrm{~cm}-1$ attributed to symmetric $\mathrm{C}-\mathrm{O}$ vibration associated with a $\mathrm{C}-\mathrm{O}-\mathrm{SO} 3$ (Camara, 2011) or the $\mathrm{C}-\mathrm{OH}$, which disappeared after synthesis of Pt-NPs. This specified the involvement of sulfate or hydroxyl groups in the reduction process of Pt-NPs. In addition, the peaks at $3658.4 \mathrm{~cm}-1$ (OH stretching) and $2981 \mathrm{~cm}-1$ (CH stretching) were also detected. After reduction of $\mathrm{H}_{2} \mathrm{PtCl}_{6}$ the decreases in intensity at 3662.4 $\mathrm{cm}-1$ imply the involvement of the $\mathrm{OH}$ groups in the reduction process. This is further confirmed with a reduction in $\mathrm{pH}$ of solution during the reaction. The above peaks generally occur in polysaccharides indicating the participation of sulfated polysaccharides in the synthesis of Pt NPs. 
The peaks at $1465 \mathrm{~cm}-1$ indicate the $\mathrm{C}-\mathrm{C}$ groups derived from aromatic rings that are present in the ocimum sanctum aqueous extract. Another peak at $1593 \mathrm{~cm}-1$ is attributed to the stretching vibration of (NH) $\mathrm{C}=\mathrm{O}$ group that is characteristic of proteins shifted from $1463 \mathrm{~cm}-1$ and became shorter after synthesis of Pt$\mathrm{NPs}$, indicating a member of $(\mathrm{NH}) \mathrm{C}=\mathrm{O}$ group within the cage of cyclic peptides is involved in synthesizing and capping the nanoparticles. In the FTIR spectrum of ocimum sanctum, PtNPs were formed. The additional peaks at 2339, 1385, 1075, and $957 \mathrm{~cm}^{-1}$ are related to PtNPs. We propose the presence of vander Waals forces of interaction between nitrogen and oxygen atoms in bio-compounds present in ocimum sanctum and PtNPs. Therefore, the FT-IR results imply that the PtNPs were successfully synthesized and capped with biocompounds present in ocimum sanctum extract by green method.

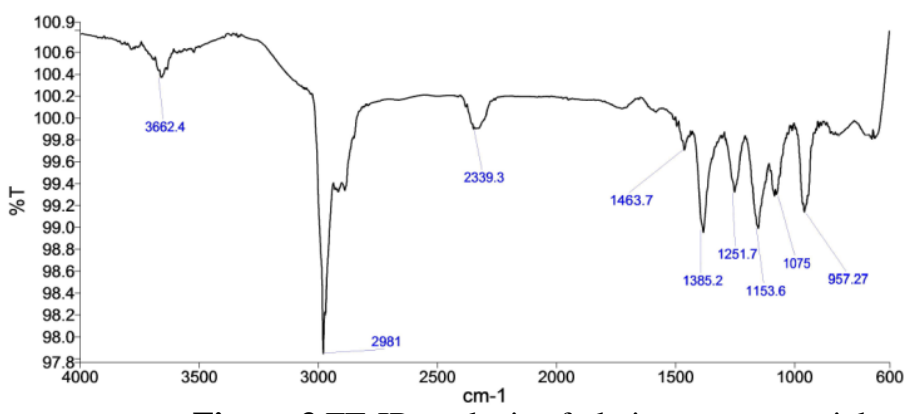

\subsection{X-Ray Diffraction}

Figure 3 FT-IR analysis of platinum nanoparticle.

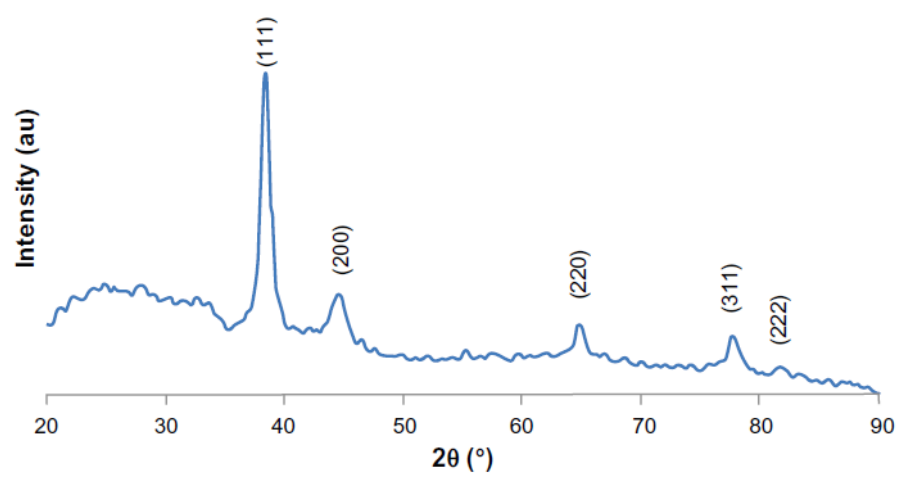

Figure 4 XRD pattern for platinum nanoparticles

The XRD pattern shows four intense peaks in the whole spectrum of $2 \theta$ values ranging from 25 to 60 . The XRD spectrum compared with the standard confirmed spectrum of platinum particles formed in the present experiments were in the form of nanocrystals as evidenced by the peaks at $2 \theta$ values of $38.14^{\circ}, 53.05^{\circ}, 66.04^{\circ}$, and $77.17^{\circ}$ corresponding to $111,200,300$, and 311 planes for platinum respectively. The value of pure platinum lattice constant has been estimated to be $\alpha=4.081$, a value that is consistent with $\alpha=4.0862 \AA$ reported by the JCPDS file no. 4-0783. This estimation confirmed the hypothesis of particle monocrystallinity. The sharpening of the peaks clearly indicates that the particles were in the nanoregime [10] reported the size of platinum nanocrystallites as estimated from the full width at half maximum of the (111) peak of platinum using the Scherrer formula was $20-60 \mathrm{~nm}$. Moreover, two small insignificant impurity peaks were observed at $60^{\circ}$ and $70^{\circ}$ which may be attributed to other organic substances in culture supernatant. XRD pattern clearly illustrates that the silver nanoparticles formed in this present synthesis were crystalline in nature. The lattice constant calculated from this pattern was $\alpha=4.086 \AA$ and the data obtained was matched with the database of Joint Committee on Powder Diffraction Standards (JCPDS) file No. 04-0783.

\subsection{Scanning Electron Microscopy}

The SEM image provided the morphology and size of the platinum nanoparticles. SEM image showed individual platinum nanoparticles as well as a number of aggregates. The morphology of the platinum nanoparticles was aggregated into larger irregular structure with no well defined morphology (Figure 5). 

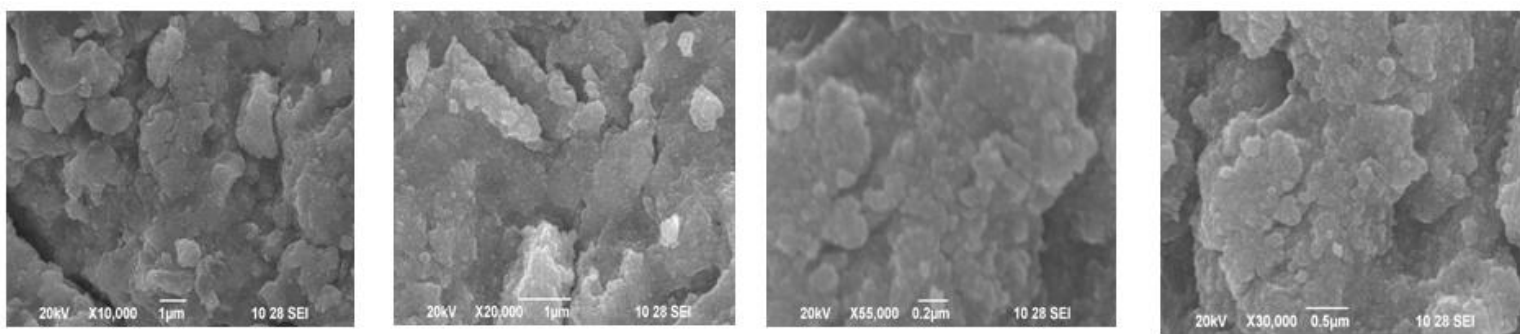

Figure 5 SEM images of the synthesized PtNPs at different magnifications.

\subsection{Transmission Electron Microscopy (Tem) Analysis}

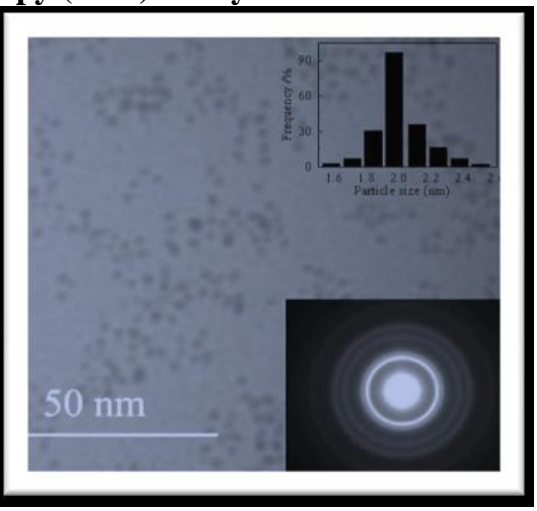

Figure 6 TEM images of the platinum nanoparticles

TEM image revealed well-dispersed platinum nanoparticles with a narrow size distribution from $1.6 \mathrm{~nm}$ to 2.5 $\mathrm{nm}$ (top-right inset). The average diameter of Pt nanoparticles was $2.0 \mathrm{~nm}$ (Figure 6).

\section{Conclusion}

Nanoparticle having one or more dimensions of the order of $100 \mathrm{~nm}$ or less have attracted considerable attraction due to their unusual and fascinating properties, with various applications, over their bulk counterparts. Currently, a large number of physical, chemical, biological, and hybrid methods are available to synthesize different types of nanoparticles. The biosynthesis of nanoparticles by plants is thought to be clean, nontoxic, and environmentally acceptable "green chemistry" procedures.

Platinum nanoparticles are enjoying increasing applications in organic catalysis, fuel cells, hydrogen storage, electrocatalysis, and bioelectronics. A convenient way to produce platinum nanoparticles is the reduction of platinum halides or anionic platinum chloride precursors with alcohols, $\mathrm{NaBH}_{4}$, sodium citrate, or $\mathrm{H}_{2}$ in the presence of surface stabilizers such as polyelectrolyte, surfactants, glucose oxidase and others.

The leaf extract of Ocimum sanctum was used as a reducing agent for the synthesis of platinum nanoparticles from an aqueous chloroplatinic acid $\left(\mathrm{H}_{2} \mathrm{PtCl}_{6} \cdot 6 \mathrm{H}_{2} \mathrm{O}\right)$. A greater conversion of platinum ions to nanoparticles was achieved by employing a Tulsi leaf broth with a reaction temperature of $100{ }^{\circ} \mathrm{C}$. The successful formation of platinum nanoparticles has been confirmed by UV-Vis spectro photometer, Fourier Transform Infrared Spectroscopy (FTIR), X-Ray Diffraction (XRD), Scanning Electron Microscopy (SEM) and Transmission Electron Microscopy (TEM).

It is evident from scanning electron microscopy that the reduced platinum particles were found as aggregates with irregular shape. FTIR studies confirmed the presence of platinum nanoparticles which may responsible for reduction of platinum ions to platinum nanoparticles, yet further research is needed in this area to explore the possible biomolecules responsible for the bioreduction process. The XRD pattern revealed the complex crystalline nature of platinum nanoparticle. In this method there is no need to use high pressure, energy, temperature and toxic chemicals in case of chemical and physical method. These nanoparticles may have a great application in the field of pharmacological and other industries. This environmentally friendly method of biological platinum nanoparticles production increases the rates of synthesis faster which can potentially be used in water electrolysis applications. It is cost effective and simple method for production of platinum nanoparticles compared to physical and chemical methods. Platinum nanoparticles are promising material for future cancer therapy. Platinum Nanoparticles used to Prevent Air Pollution in the environment. 


\section{References}

[1]. Baker, C., Pradhan, A., Pakstis, L., Pochan, D.J. and Shah, S.I., (2005) 'Synthesis and Antibacterial Properties of Silver Nanoparticles', J. Nanosci. Nanotechnol 5, 224-249.

[2]. Shankar SS, Rai A, Ahmad A, Sastry M. Rapid synthesis of Au, Ag, and bimetallic Au core Ag shell nanoparticles using Neem (Azadirachta indica) leaf broth. J Colloid Interface Sci 2004;275:496-502.

[3]. Chul Jae Lee., Mohammad Rezaul Karim., Vasudevan, T., Hee Jin Kim., Raushan, K., Maeng Joon Jung., Dong Yeub Kim. and Mu Sang Lee. (2010) 'A Comparison Method of Silver Nanoparticles Prepared by the Gamma Irradiation and in situ Reduction Methods', Bull. Korean Chem. Soc. Vol.31

[4]. Song, J. Y.; Kwon, E. Y.; Kim, B. S. Biological synthesis of platinum nanoparticles using Diopyros kaki leaf extract. Bioprocess Biosyst. Eng. 2010, 33 (1), 159-164

[5]. Coccia, F. Tonucci, L. Bosco, D. Bressan, M. d'Alessandro, N. One pot synthesis of lignin-stabilized platinum and palladium nanoparticles and their catalytic behaviours in oxidation and reduction reactions, Green Chem. 2012, 14 (4), 1073-1078

[6]. Soundarrajan, C. Sankari, A. Dhandapani, P. Maruthamuthu, S. Ravichandran, S. Sozhan, G. Palaniswamy, N. Rapid biological synthesis of platinum nanoparticles using Ocimum sanctum for water electrolysis applications. Bioprocess Biosyst. Eng. 2012 , 35 (5), $827-833$

[7]. S. Mondal, B. R. Mirdha, and S. C. Mahapatra, "The science behind sacredness of Tulsi (Ocimum sanctum linn.)," Indian Journal of Physiology and Pharmacology, vol. 53, no. 4, pp. 291-306, 2009

[8]. Jayshree Nellore, 1 Cynthia Pauline, 1 and Kanchana Amarnath, Bacopa monnieri Phytochemicals Mediated Synthesis of Platinum Nanoparticles and Its Neurorescue Effect on 1-Methyl 4-Phenyl 1,2,3,6 Tetrahydropyridine-Induced Experimental Parkinsonism in Zebrafish, Journal of Neurodegenerative Diseases Volume 2013, pp 1-8

[9]. Paul mulvaney, surface plasmon spectroscopy of nanosized metal particles, 1996, vol.12, 788-800

[10]. Manish Dubey, seema Bhadauria, kanhiya mahour, r.k gautam formation of highly bioactive nanoparticles and their antibacterial activity pharmacologyonline 2009 , vol 3, 437-446 